\title{
An interview with Gary D Hammer, MD, PhD
}

\author{
Gary D Hammer*,1 \\ ${ }^{1}$ Endocrine Oncology Program, Rogel Cancer Center, University of Michigan, Ann Arbor, Michigan 48109, USA \\ *Author for correspondence: ghammer@umich.edu
}

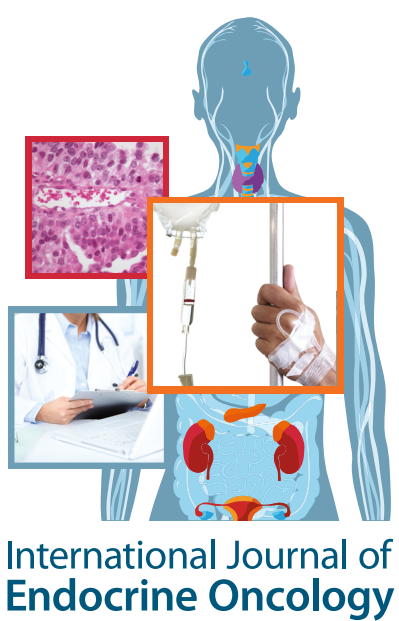

Dr Hammer is the cofounder of Millendo Therapeutics (NASDAQ, MLND, MI, USA) and the founder of Vasaragen (MI, USA), two biotechnology companies focused on rare endocrine diseases. Hammer is also an employee of the University of Michigan.

First draft submitted: 2 December 2019; Accepted for publication: 2 December 2019; Published online: 23 December 2019

Keywords: adrenal • adrenocortical carcinoma • cortisol • endocrine oncology • homeostasis $\bullet$ hormone $\bullet$ stem cell - steroid

\section{Could you please introduce yourself \& provide a brief summary of your career to date?}

My name is Gary Hammer, I am the Director of the Endocrine Oncology Program at the University of Michigan Rogel Cancer Center (MI, USA). I also serve as the President-Elect of the Endocrine Society - the world's largest professional organization of endocrinologists and scientists.

My interest in endocrinology and the adrenal gland was sparked by my early interest in the biology of stress. After working on the neurobiology of fear as an undergraduate student in the Laboratory of Bruce Kapp at the University of Vermont (VT, USA), I obtained my MD and PhD at Tufts University School of Medicine (MA, USA), where I studied the regulation of the ACTH (adrenocorticotropin) gene POMC (proopiomelanocortin) in the pituitary under the mentorship of Malcolm Low. I then proceeded to the University of California in San Francisco (CA, USA), where I completed my residency in Internal Medicine, fellowship in Endocrinology and then a postdoctoral fellowship in molecular endocrinology and development in the laboratory of Holly Ingraham. It was here that I began exploring the signaling pathways and transcriptional mechanisms that regulate adrenal cortical growth and differentiation.

My interest in endocrine cancers arose from my training with Ingraham in understanding the regulation, development and homeostasis of the adrenal gland, which intersected with my continued clinical interest in the diseases of the hypothalamic-pituitary-adrenal axis. In particular, I had always been fascinated with the diseases of the pituitary and adrenal, specifically those that involved hormonal hypofunction or hyperfunction. With all endocrine systems being predicated on feed-forward activation pathways and feedback inhibitory pathways, diseases of the hypothalamic-pituitary-adrenal ultimately manifest in diseases of the adrenal gland. I began to cultivate an increasing fascination with adrenal hypoplasia, hyperplasia, neoplasia and adrenal cancer. These disorders serve as the intersection of endocrine organ form and function. As such, endocrine neoplasia is the unique convergence of endocrinology and oncology.

In 1999, I left the University of California in San Francisco (14 years after graduating college). I came to the University of Michigan to be part of a resurgence or reinvestment in the growth of endocrine oncology as a bona fide discipline. Bo Schembechler, famed University of Michigan football coach, lost his wife Millie to adrenocortical carcinoma a few years before my arrival. He raised significant funds to reinvigorate the adrenal team at the University of Michigan. With the support of Max Wicha, the founding Director of the University of Michigan Rogel Cancer Center, I began my tenure in the first and only faculty post of my career. I became the inaugural Director of the Endocrine Oncology Program at the Rogel Cancer Center a few years later. We have developed a multidisciplinary team of scientists and clinicians to advance science, provide care and build an international network that advocates, educates and collaborates to find effective treatments for adrenal cancer and associated endocrine malignancies.

Future Medicine 


\section{Could you please provide a brief introduction to the research going on in your lab?}

My laboratory initially focused on the transcriptional signaling pathways that are engaged in adrenal cortical growth and development. We found ourselves spending more and more time trying to define the nature of the adrenal cortical stem and progenitor cell population. As we and others began to define this population, it became clear that the same signaling pathways and transcription factors that engaged in normal homeostasis were disrupted in diseases of hypoplasia, hyperplasia and cancer. That led us into the translational space of trying to define new molecular signatures of adrenal cortical carcinoma, which has led us to the development of novel therapeutics for adrenal cancer.

Our group now explores the signaling and transcriptional control of stem cell lineages in the adrenal cortex. Applying molecular genetics, ourselves and others began to appreciate the centripetal and radial nature that defined the life of an adrenal cell. Studies in genetically engineered mouse models gave critical insights. Multipotent undifferentiated adrenocortical stem cells that do not secrete any steroids, reside in the peripheral cortex and serve as stem cells endowed with presumed selfrenewal and the ability to give rise to the differentiated lineages of the cortex. These cells live their whole life from 'out-to-in' - first as undifferentiated stem cells, then as peripheral angiotensin-responsive mineralocorticoid producing cells of the glomerulosa, before they are displaced centripetally and differentiate in an ACTH-responsive fasciculata cell. After further differentiating into the central androgen producing reticularis cell, the cell dies an apoptotic death at the corticomedullary boundary.

Interestingly, we and others learned that the adrenal capsule is a critical component of the adrenal stem cell niche and participates in a signaling relay, whereby the capsule communicates with the cortical stem cells and the cortical stem cells communicate with the capsule. The interplay between these paracrine signals and the endocrine aspects of adrenal differentiation (steroidogenesis) are increasingly realized as paramount.

The adrenal and gonad share a common embryologic origin - the adrenogonadal primordia that undergo developmental bifurcation. This leads to separate adrenal cortices that respond to ACTH and gonads that respond to the gonadotropins luteinizing hormone and follicular stimulating hormone. Our lab and many others have defined unique genes that specify these milestones. Early studies from our laboratory discovered that the gene inhibin (INHBA) serves to maintain the steroidogenic fate of the adrenal cortex. Without inhibin, the adrenocortical stem cell will differentiate into both ovarian lineages - theca and granulosa cells - organized in follicular structures.

These early studies pushed us to understand more deeply the pathways engaged in stem cell selfrenewal versus steroidogenic differentiation. For example, loss of function mutations in $D A X-1$ results in cytomegalic X-linked adrenal hypoplasia in male infants. Occasionally, however, in two male twins with the same mutation, one boy may develop adrenal failure in infancy, but the brother will not develop failure until late in puberty. At times, patients with the late onset disease may initially develop a hyperactive adrenal cortex (i.e., glucocorticoid excess of Cushing syndrome) before ultimately developing failure. It was our collaborative work with Larry Jameson (University of Pennsylvania, PA, USA) that enabled us to define the role of Dax-1 as a stem cell maintenance factor. When DAX-1 was mutated in mice, we were able to demonstrate rapid differentiation of the cortex at the expense of late adrenal failure, presumably due to stem cell exhaustion. Additional studies followed on the roles of paracrine Wnt (a portmanteau of Wingless and Int-1) and Shh (sonic hedgehog) signaling in the cortex, specifically maintenance of the stem cell pool. These studies provide increasing clarity and molecular tools, which allow continued refinement of our understanding of normal homeostatic maintenance of the adrenal cortex.

As our clinical Endocrine Neoplasia Program grew, we began studying the genomic underpinnings of adreal neoplasia and learned that developmental homeostatic genes that we had been studying for a decade or more, were also implicated in adrenal cancer. The lab then began to explore how homeostatic stem cell pathways were exploited by adrenal cancer. These two lines of investigation have been the basic cornerstones of our lab, which has fueled our translational efforts to develop new therapeutics for adrenal cancer over the last 5-10 years.

\section{How have you seen the adrenal cancer field develop over the past 15 years?}

In 2003, we held the first international symposium on adrenal cancer at the University of Michigan. We invited approximately 20 people from around the world - the few labs and clinicians who were doing anything related to adrenal neoplasia. Our aim was to write a consensus statement and guidelines for the management of adrenal cancer.

At the time we knew very little about the disease. We also did not know each other well, and therefore did not trust each other as completely as we needed to work together. As such, we could not write any meaningful guidelines until we dug deeper into the biology of this disease, but, on that day, we agreed to work together in a 
collaborative manner to develop new research protocols and large platforms of discovery to help us ultimately push forward with treatments and care guidelines.

It took us 15 years. We had to go back and understand the science and work together to engage in discovery work, disease-oriented and patient-oriented research. In rare diseases, samples are precious. This is true of most endocrine neoplasia and certainly adrenal cancer, where less than 2000 new cases are diagnosed per year in the USA and Europe combined. We realized that we could combine our resources to engage in research, both basic and clinical. We would leverage our combined laboratories with funding agencies for large collaborative grants and we would leverage our network for clinical trials that could not easily be funded outside of Big Pharma. This strategy has been remarkably successful. The emerging adrenal cancer community spent the last two decades developing two large platforms of engagement. One was born out of that initial meeting in 2003, the European Network for the Study of Adrenal Tumors. The second is the American Australian Asian Adrenal Alliance (A5). I am happy to say that last year, the first guidelines for the management of adrenocortical carcinoma were published in the European Journal of Endocrinology - written by European Network for the Study of Adrenal Tumors and endorsed by A5.

In oncology, as targeted therapies take center stage, it is quickly becoming essential to define predictors of response. It is critical to understand the genetic and genomic signatures of responders, as well as the relationship between a biomarker and responsiveness. Just because a genetic marker is present in cancer does not mean that it is therapeutically targetable. Most of oncology is heading in this direction. We aim to understand what subset of patients respond to drug X. For a drug to be approved with a biomarker predictor, the predictor must be validated and developed as a clinical diagnostic test, which often needs to be approved by the US FDA. For example, to get a genetic test approved and linked to a new therapy being developed, it costs somewhere between US $\$ 5$ million and US $\$ 20$ million, just to have the test approved. It is a lot of money to get a drug approved for a common disease, let alone a rare disease. For the rare diseases, the science needs to be tight.

More recently, disease consortia and patient advocacy groups are partnering to effectively mitigate risk and enhance enthusiasm for industry engagement. This is precisely how the adrenal cancer community successfully engaged and partnered with funding agencies and industry leaders to execute large genomic projects and international clinical trials in adrenal cancer.

Doctors, scientists and industry must work together to ultimately benefit the patients. And, quite frankly, we are seeing more and more academic centers cooperating with and working with industry - either spinning out their own companies or working with companies to enact change.

\section{What are the current treatments available for adrenocortical carcinoma?}

The only FDA-approved drug for adrenal cancer is mitotane, which is a derivative of the pesticide dichlorodiphenyltrichloroethane (DDT). When toxicity studies were finally performed on mitotane in the late 1960 s and early 1970 s, it became clear that it was toxic to the dog adrenal gland. Those initial studies single-handedly ignited the environmental movement upon which the Environmental Protection Agency was founded and DDT was banned from the Earth. Despite that, its derivative o,p'-DDD (mitotane or 2,4'-dichlorodiphenyl)dichloroethane, 1-(2chlorophenyl)-1-(4-chlorophenyl)-2,2-dichloroethane), which was also shown to be toxic to the dog adrenal gland, became the first and only drug to treat adrenal cortical carcinoma. Although it is difficult to tolerate, it is indeed effective in some patients and strategies have been developed to limit toxicity and to maximize benefit.

The first project that aimed to identify effective therapies other than mitotane was born out of that first 2003 meeting. The First International Randomized Trial in Locally Advanced and Metastatic Adrenocortical Carcinoma Treatment (FIRM-ACT) was the first ever randomized trial for adrenal cancer. It enrolled more than 300 patients, which for an adrenal cancer trial is spectacular. It proved that the global adrenal community could work together and engage. It also showed that the combination of Etoposide, Doxorubicin, Cisplatin (referred to as EDP) and mitotane was an appropriate first-line therapy for this disease.

EDP, with or without mitotane, is used often by the adrenal cancer community for the treatment of metastatic adrenal cancer. Those are the two therapies, but the response rates are still rather poor: less than $23 \%$ response rates for EDP, $20 \%$ for mitotane alone. For this reason, new research, collaborative research and new therapies are desired. 
What do you see as the biggest challenges facing the development of novel therapeutics \& what are you doing to overcome these challenges?

One of the biggest challenges for the development of novel therapeutics has been the engagement of pharma, where the return on investment is not as high as in a common disease. The risk-benefit fulcrum has shifted a bit over the last 10 years, such that big pharma is not as risk averse to rare diseases. This is a welcome change and partly predicated on changing the regulatory climate that incentivizes rare disease investment. As such, the investment houses (i.e., Angel Funds and Venture Capital firms) have become less averse to rare diseases as well - if the scientific thesis is sound and a modest return-on-investment can be actualized.

It was not until we really delved deeper into the science and proved how critical certain signaling pathways were in the disease biology that the pharmaceutical industry came to the table and participated in relatively large (large for a rare disease, that is) Phase I, Phase II and Phase III trials for adrenal cancer. Moreover, as it became clear that we could engage and leverage our cooperative groups in both the scientific discovery, which we had together amassed and in large clinical trials, the investment thesis became viable for the pharmaceutical community.

Take ALK4 (activin-like kinase receptor-4) in non-small-cell lung cancer as an example. ALK4 is mutated in approximately $3 \%$ of lung cancer, but nonetheless, ALK inhibitors were approved for non-small-cell lung cancer harboring the target mutation. FDA approval along with the approval of Gleevec for gastrointestinal stromal tumors helped turn the tide. Drug companies have become much less risk averse and actually interested in going after rare diseases and rare oncologic indications, partly due to the incentives to develop therapies for these diseases and partly because patient advocates are so much more engaged and pushing the agenda for their own diseases.

In addition, it is often easier to achieve approval for a rare indication, because the FDA and the government have provided certain benefits for getting drugs in the pipeline for rare diseases. For example, 'breakthrough status' and 'orphan disease status' where a company will be granted extra 'time-on-runway' for patent life.

\section{Throughout your career, there have been several technological advances. What would you say has been the biggest technological innovation in improving your research?}

We can now utilize single cell genomic technologies, which allow us to probe the mutational landscape and genetic perturbations that occur in the individual cancer cells over time. Such genomic complexity and heterogeneity can seem limitless where cancer has an indefatigable ability to change, grow and morph over time. Our charge is to stay one step ahead of this cellular arms race.

In the diagnostic arena, one of the biggest challenges with adrenal tumors is that not all of them are cancerous. Up to 20 million adrenal tumors are detected every year in the USA on CT scans. But, only 1000 of these masses are malignant adrenocortical carcinoma. So how do we distinguish them on a CT scan alone? We have historically removed many tumors that in retrospect did not need to be removed. But, as they cannot be defined preoperatively as $100 \%$ benign, they are resected. Now, the field of steroidomics is evolving and we understand how steroids are metabolized differently in different tissues. This means scientists can now distinguish benign from malignant cancers by looking at the adrenal steroid profile in urine or blood alone, to both diagnose cancer and assess its return before something is visible on a CT scan. Moreover, cell and DNA/RNA detection capabilities are evolving, it is now possible to detect cancer cells or DNA/RNA in the blood before the patient has evidence of disease on CT scans. Ideally, these emerging technologies will help get the right patient the right therapy at the right time. That is the goal.

\section{What was the inspiration surrounding the development of Millendo Therapeutics?}

This is a personal story. Millendo (MI, USA) was conceptualized and founded by a patient of mine, Raili, who was also the wife of a biochemistry professor and colleague here at the University of Michigan. Raili was diagnosed with adrenal cancer and was being treated in our clinic.

Raili had worked for decades in the pharmaceutical industry. She became quite frustrated at our inability to provide more care than mitotane or cytotoxic chemotherapy. Riley ran into a former colleague from 30 years ago who reminded her of a class of drugs that were used to lower cholesterol levels and decrease the formation of atherosclerotic plaque, as well as blocking the esterification of cholesterol. It turns out, they had a toxic effect on the adrenal gland and were therefore abandoned as statins came to market for cholesterol management.

We started thinking that cholesterol ester itself, which is particularly enriched in the adrenals because it serves as the precursor of all steroid hormones, may serve as a target for a therapeutic agent for adrenal cancer. 
Raili began to study this hypothesis in my own laboratory and that of her husband's. It was clear that inhibiting cholesterol esterification was toxic to the adrenal cortical cell. We figured out the mechanism that resulted in its toxicity: endoplasmic reticulum stress due to the high levels of free cholesterol. Raili then involved me in forming a company called Millendo to repurpose this class of compounds for adrenal cancer. She single-handedly raised US\$16 million and founded Millendo with me and CEO Julia Owens. Currently, Millendo has paused its endocrine oncology indication, but it is still engaged in the use of this class of drugs on the steroid excess that occurs in adrenal neoplasia.

Millendo taught me the importance of an academic-industry partnership. And so, predicated on work we had done defining a critical role of the growth factor IGF2 (insulin-like growth factor 2) in adrenal cancer, I founded the company Vasaragen (MI, USA). Our genomic studies with colleague Tom Giordano, MD, PhD (University of Michigan, MI, USA), led us to the conclusion that the IGF-signaling pathway is critically engaged in the adrenal cancer phenotype. There have been many studies on the IGF2 pathway in adrenal cancer. It was one of the first pathways we were able to convince Big Pharma to invest in, with three clinical trials for adrenal cancer including a Phase I, Phase II and Phase III trials, using different drugs that inhibited the IGF pathway. Although the drugs worked, they were only effective in a small subset of patients in each trial. Ten to fifteen years ago, it was very difficult to get drugs approved when they only worked in a small percentage of treated patients. This was a particular challenge, as approval was historically granted only if overall survival (rather than progression-free survival) was increased in the drug-treated group versus the placebo group. This is difficult to achieve when only a small percentage of treated patients respond to treatment.

It is now clear that oncology patients need to be preselected for targeted treatments when they have a diagnostic or predictive marker. It was not until after we engaged in the large consortium-based platforms of discovery (i.e. The Cancer Genome Atlas) that we have been able to understand the signatures that presumably predict response to individual therapies for adrenal cancer. Out of that Vasaragen was born, which aims to get targeted therapies to the patients that have the right genomic signature for a given targeted molecule (precision medicine).

Our goal is to capitalize on our understanding of the genomic footprints of adrenal cancer, the knowledge that we can now separate adrenal cancer into different genomic subsets for which different agents are predicted to respond (in some cases, we already have data that they do respond). The goal is to leverage this knowledge in order to repurpose drugs that have shelved because they did not reach the placebo-controlled benchmark. In a sense, we are resurrecting prior molecules that have been tested and failed due to inadequate patient selection. We can now define the subsets of patients that have the right genetic predictor of response.

This is the future of medicine: to get the right drug to the right patient at the right time.

Financial \& competing interests disclosure

The authors have no relevant affiliations or financial involvement with any organization or entity with a financial interest in or financial conflict with the subject matter or materials discussed in the manuscript. This includes employment, consultancies, honoraria, stock ownership or options, expert testimony, grants or patents received or pending, or royalties.

No writing assistance was utilized in the production of this manuscript.

\section{Disclaimer}

The opinions expressed in this interview are those of the interviewee and do not necessarily reflect the views of Future Medicine Ltd (Future Science Ltd).

\section{Open access}

This work is licensed under the Attribution-NonCommercial-NoDerivatives 4.0 Unported License. To view a copy of this license, visit http://creativecommons.org/licenses/by-nc-nd/4.0/ 